$10.1117 / 2.1201211 .004541$

\title{
Compact, high-efficiency sunlight harvesting
}

Fabian Duerr, Pablo Benítez, Juan Carlos Miñano, Youri Meuret, and Hugo Thienpont

A new free-form optics design method could unleash the full potential of tracking integrated solar concentrators

Concentrating photovoltaic (CPV) systems use optics to concentrate sunlight onto solar cells. By increasing the concentration of light that reaches the solar cell, CPV systems allow the area of the solar cell to be reduced. Solar cells are made of expensive semiconductor material, and CPV systems consist of inexpensive mirrors or lenses. Thus, a CPV module-consisting of the CPV system, and the cell-can be cheaper than the solar cell alone, but equally efficient. High-efficiency multi-junction solar cells can boost the conversion efficiency of CPV modules beyond $30 \%$, but their expense means they require a high concentration of light $(>400 \times)$ to be economically viable. Achieving this level of concentration normally requires dual-axis tracking of the sun's diurnal and seasonal movements.

Most CPV manufacturers work with very large modules and pedestal-mounted dual-axis trackers: see Figure 1. These systems are suitable for utility-scale power plants, but are less adequate for providing power to mid-scale or smaller operations. In contrast, photovoltaic (PV) modules with single-axis trackers are already in use on flat rooftops. Currently, CPV systems designed for single-axis trackers are limited to a concentration of about $300 \times$ for polar alignment, where the tracker axis equals the Earth's axis of rotation. ${ }^{1}$ This concentration is not sufficient for economic use of multi-junction solar cells.

Existing CPV system designs vary, but almost all treat the CPV modules and the external trackers separately: see Figure 2(a). In contrast, our approach integrates part of the external solar tracking functionality into the CPV module: ${ }^{2}$ see Figure 2(b). The laterally-moving optics arrays are mounted on a polar-aligned single-axis tracker, and combine the concentration and steering of the incident sun light. Tracking integration can be used to reduce the external tracking effort in favor of compact installation size $^{3-5}$ or to fine-tune the total tracking functionality, allowing coarse external solar tracking. ${ }^{6}$

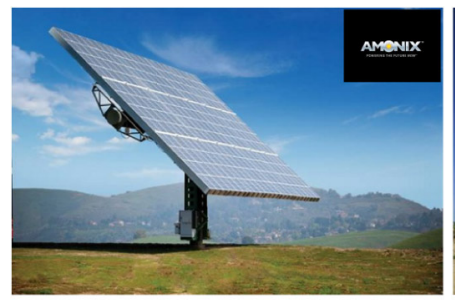

(a)

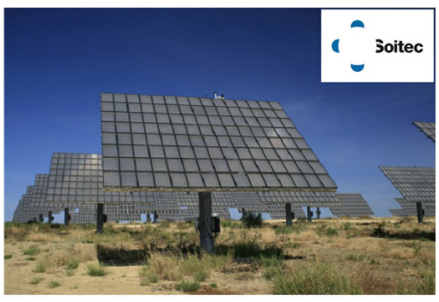

(b)
Figure 1. Concentrating photovoltaic installations marketed by (a) Amonix and (b) Soitec Concentrix. (Amonix photo by Jeff Aubin. Concectrix photo reprinted with permission from Concentrix.)

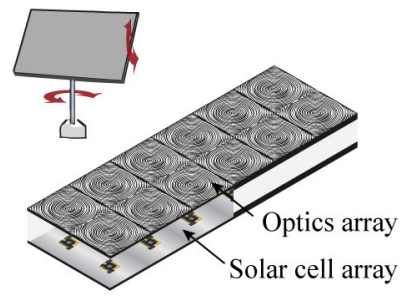

(a)

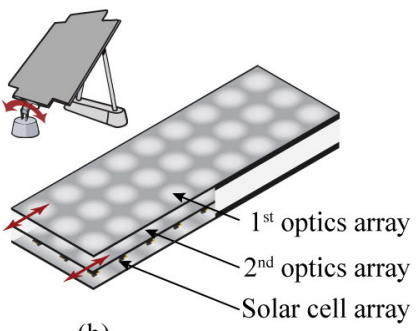

(b)
Figure 2. (a) Schematic assembly of a conventional concentrating photovoltaic $(C P V)$ module for pedestal-mounted dual-axis trackers, and (b) our tracking integrated CPV module for polar-aligned single-axis trackers $(b)$.

While a benefit of dual-axis trackers over fixed installationswhich allow for the modules to always be pointed at the sun-is the increased yearly insolation, comparing the potential annual energy yield for polar-aligned single-axis trackers with dual axis trackers shows only moderate differences. ${ }^{7}$ The tracking integration of our single-axis system covers an angular range of $\pm 24^{\circ}$ for incident direction vectors (pointing at the sun) within a single plane. If symmetry is considered, it is evident that rotational symmetric lenses are not an optimal solution. We developed a new design algorithm, ${ }^{8}$ based on the simultaneous multiple 
surface (SMS) design method in 3D. ${ }^{9}$ This algorithm allows the simultaneous calculation of two optical surfaces, focusing two off-axis ray sets. The free-form lenses designed from this method have rectangular apertures. Our free-form lens design outperforms its rotational symmetric counterpart, exceeding the desired $400 \times$ concentration for a polar-aligned single-axis tracker: see Figure 3.

Figure 4 shows our optical design has two distinct features: a wide field of view, and a clear separation between the two optical surfaces. We have demonstrated with the new analytic design algorithm that it is possible to couple more than two ray sets with only two lens surfaces for such configurations. ${ }^{10}$ This can only be achieved if different ray sets use different portions of the lens surfaces. The convergence points, which are characterized by on- and off-axis rays sharing identical points and normal vectors on each lens profile, are key in our design method: see Figure 4 . These were first introduced in the design of aspheric (a)

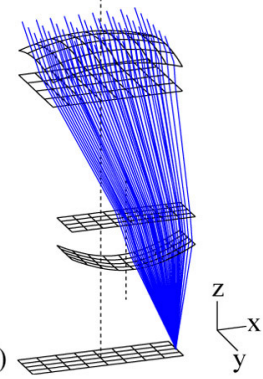

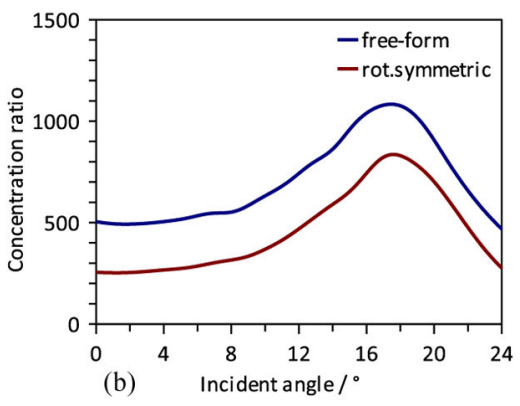

(b)

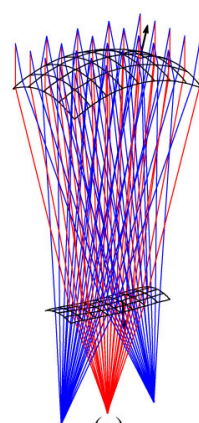

(a)

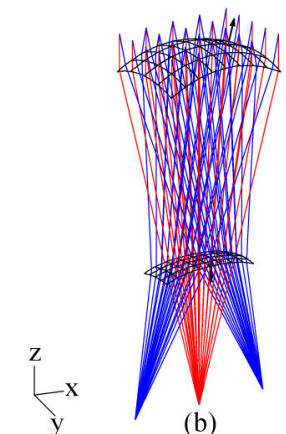

(b)
Figure 5. Ray tracing results for two exemplary solutions for calculated free-form lenses: (a) a meniscus and (b) a biconvex lens.

V-groove reflectors. ${ }^{11,12}$ We used Fermat's principle to deduce a set of functional differential equations fully describing the entire optical system. Our presented general analytic solution makes it possible to calculate the lens profiles up to $20^{\text {th }}$ order Taylor series about the convergence points. ${ }^{13}$

We generalized the 2D analytic optics design method to the 3D case. ${ }^{14}$ We used Fermat's principle to derive additional sets of functional differential equations, which made it possible to calculate the Taylor series functions describing the free-form lens surfaces with more than 100 coefficients. Two exemplary calculated solutions are a meniscus and a biconvex lens: see Figure 5. We have previously demonstrated the potential use of this new free-form optics design method for imaging applications with a high aspect ratio. 15

In summary, we have presented the concept of tracking integration and indicated its potential to open small to mid-scale installation markets for $\mathrm{CPV}$ due to the strong reduction of the external solar tracking effort. Our new free-form design method shows a way to realize the full potential of the optical system. Next, we intend to apply the analytic design method to our tracking integration optics and work on a first demonstrator.

Our work is supported in part by the Flanders Research Foundation (FWO Vlaanderen) that provides PhD funding (grant FWOTM510) for Fabian Duerr, and in part by the Belgian Science Policy Office under its 'Interuniversity Attraction Pole' programme. Funding from $V U B$ 's 'Industrial Research Funding' (IOF) and 'Methusalem' funding programmes, and the VUB Research Council (OZR) is also gratefully acknowledged.

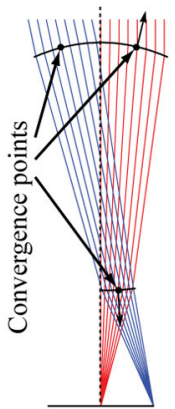

(b) (c)

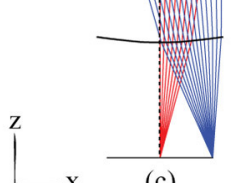

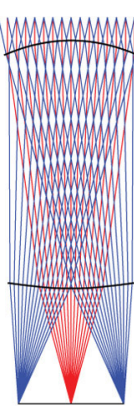

(d) (a)

Figure 4. Simplified 2D SMS reference design illustrating the wide field view, and separation of optical surfaces of our design. (a)-(c) Steps in coupling an additional on-axis ray set. (d) Final ray paths. 


\section{Author Information}

Fabian Duerr, Youri Meuret, and Hugo Thienpont

Brussels Photonics Team (B-Phot)

Department of Applied Physics and Photonics

Free University (VUB)

Brussels, Belgium

Fabian Duerr is working towards a PhD. His research is on concentrating photovoltaics, with a focus on direct design methods for free-form optics.

Youri Meuret is a postdoctoral researcher. He has published several papers and collaborated with many companies in the domain of optical modeling and design.

Hugo Thienpont chairs the Applied Physics and Photonics Department and is director of its photonics research group. He is also a full professor in the Faculty of Engineering at VUB.

\section{Pablo Benítez and Juan Carlos Miñano}

Center for Energy Efficiency and Smart Buildings (CeDInt)

Technical University of Madrid (UPM)

Madrid, Spain

and

LPI

Altadena, CA

Pablo Benítez is associate professor at UPM and associate scientist at LPI. He researches optical design, predominantly in concentration photovoltaics, solid-state lighting, and free-form imaging. He co-authored the reference book Nonimaging Optics (Elsevier, 2004) and is co-inventor of the simultaneous multiple surfaces optical design method in 2D and 3D.

Juan Carlos Miñano is a full professor at UPM and also coauthored the book Nonimaging Optics (Elsevier, 2004). In 2010 he received the A. E. Conrady Award from SPIE in recognition of his contribution to nonimaging optics. In addition, he is a senior scientist at LPI.

\section{References}

1. A. Mohr, T. Roth, and S.W. Glunz, BICON: high concentration PV using one-axis tracking and silicon concentrator cells, Prog. Photovolt. Res. Appl. 14 (7), pp. 663 674, 2006. doi:10.1002/pip.691

2. F. Duerr, Y. Meuret, and H. Thienpont, Tracking integration in concentrating photovoltaics using laterally moving optics, Opt. Express 19 (103), pp. A207-A218, 2011. doi:10.1364/OE.19.00A207

3. J. H. Karp, E. J. Tremblay, and J. E. Ford, Planar micro-optic solar concentrator, Opt. Express 18 (2), pp. 1122-1133, 2010. doi:10.1364/OE.18.001122

4. J. Hallas, J. Karp, E. Tremblay, and J. Ford, Lateral translation microtracking of planar micro-optic solar concentrator, Proc. SPIE 7769, p. 776904, 2010. doi:10.1117/12.860980

5. J. Hallas, K. Baker, J. Karp, E. Tremblay, and J. Ford, Two-axis solar tracking accomplished through small lateral translations, Appl. Opt. 51 (25), pp. 6117-6124, 2012. doi:10.1364/AO.51.006117

6. W. C. Sweatt, B. H. Jared, G. N. Nielson, M. Okandan, A. Filatov, M. B. Sinclair, J. L. Cruz-Campa, and A. L. Lentine, Micro-optics for high-efficiency optical performance and simplified tracking for concentrated photovoltaics (CPV), Proc. SPIE 7652, p. 765210, 2010. doi:10.1117/12.870964

7. T. Huld, T. Cebecauer, M. Šúri, and E.D. Dunlop, Analysis of one-axis tracking strategies for PV systems in Europe, Prog. Photovolt. Res. Appl. 18 (3), pp. 183-194, 2010. doi: $10.1002 /$ pip. 948

8. F. Duerr, P. Benítez, J. C. Miñano, Y. Meuret, and H. Thienpont, Integrating tracking in concentrating photovoltaics using non-rotational symmetric laterally moving optics, Proc. SPIE 8124, p. 81240M, 2011. doi:10.1117/12.893330

9. P. Benítez, J. Miñano, J. Blen, R. Mohedano, J. Chaves, O. Dross, M. Hernandez, and W. Falicoff, Simultaneous multiple surface optical design method in three dimensions, Opt. Eng. 43, p. 1489, 2004. doi:10.1117/1.1752918

10. F. Duerr, P. Benítez, J. C. Miñano, Y. Meuret, and H. Thienpont, Analytic design method for optimal imaging: coupling three ray sets using two free-form lens profiles, $\mathbf{O p t}$ Express 20 (5), pp. 5576-5585, 2012. doi:10.1364/OE.20.005576

11. D. Grabovičkić, P. Benítez, and J. Miñano, Aspheric V-groove reflector design with the SMS method in two dimensions, Opt. Express 18 (3), pp. 2515-2521, 2010. doi:10.1364/OE.18.002515

12. D. Grabovičkić, P. Benítez, and J. Miñano, Free-form V-groove reflector design with the SMS method in three dimensions, Opt. Express 19 (104), pp. A747-A756, 2011 doi:10.1364/OE.19.00A747

13. F. Duerr, P. Benítez, J. C. Miñano, Y. Meuret, and H. Thienpont, Perfect imaging of three object points with only two analytic lens surfaces in two dimensions, Proc. SPIE 8429, p. 6, 2012. doi:10.1117/12.922089

14. F. Duerr, P. Benítez, J. C. Miñano, Y. Meuret, and H. Thienpont, Analytic freeform lens design in 3D: coupling three ray sets using two lens surfaces, Opt. Express 20 (10), pp. 10839-10846, 2012. doi:10.1364/OE.20.010839

15. F. Duerr, P. Benítez, J. C. Miñano, Y. Meuret, and H. Thienpont, Analytic free-form lens design for imaging applications with high aspect ratio, Proc. SPIE 8486, p. 848609, 2012. doi:10.1117/12.929243 